\title{
Bronchodilator combinations for COPD: real hopes or a new Pandora's box?
}

\author{
Nicolas Roche ${ }^{1}$ and Pascal Chanez ${ }^{2}$
}

\begin{abstract}
Affiliations: ${ }^{1}$ Respiratory and Intensive Care Medicine, Cochin Hospital Group, AP-HP, EA2511 University Paris Descartes, Paris, and ${ }^{2}$ Respiratory Medicine APHM, INSERM U1067, Aix Marseille University, Marseille, France.
\end{abstract}

Correspondence: N. Roche, Pneumologie et Soins Intensifs Respiratoires, Groupe Hospitalier Cochin, Site Val de Grâce, 4eC, 74 Bd de Port Royal, 75005 Paris, France. E-mail: nicolas.rochedacch.aphp.fr

0

@ERSpublications

The SHINE study describes the effect of dual $v s$ single bronchodilation in COPD, but what does this mean for patients? http://ow.ly/plAHX

\section{Introduction}

Chronic obstructive pulmonary disease (COPD) is a difficult-to-treat condition with multiple outcomes with which to assess new drugs. Most treatments are symptomatic; none, at present, interfere with the natural history of the disease (except smoking cessation) and little is known about the factors able to predict a response. New anti-inflammatory agents able to modify the course of the disease may emerge, but further research is needed to assess their effects [1]. New drugs have to improve lung function and, most importantly, clinical outcomes with a demonstrated long-term safety at a reasonable cost. The crucial issues when combining bronchodilators are to investigate the magnitude of observed effects versus single agents and whether a "responding phenotype" can be identified. These questions are relevant for all the actors in the COPD field, from patients to health authorities, and will allow the definition of whether and how the new treatment approach will integrate with the current treatment guidelines.

In this issue of the European Respiratory Journal, BATEMAN et al. [2] report results of the SHINE study, which assessed QVA149, an association of indacaterol, a long-acting $\beta_{2}$-agonist (LABA) and glycopyrronium, a long-acting anticholinergic agent (LAMA), in a dry powder capsule device. This 26-week, large $(\mathrm{n}=2144)$, randomised controlled trial (RCT) in patients with moderate-to-severe COPD compared QVA149 to its monocomponents, open-labelled tiotropium and placebo, all administered once daily. QVA149 improves dyspnoea and health-related quality of life, with statistically significant superiority over placebo and tiotropium, but not over its monocomponents. QVA149 was significantly more effective than its monocomponents, but not tiotropium in terms of lung function (as measured using trough forced expiratory volume in $1 \mathrm{~s}(\mathrm{FEV} 1))$. These data extend results of RCTs evaluating QVA149 effect on lung function [3,4], short-term (14 days) cardiovascular safety [5] and long-term (6-12 months) "general" safety $[2,3]$. A study in patients with severe to very severe COPD and a history of exacerbations showed that QVA149 decreases the rate of moderate to severe exacerbations by $12 \%$ when compared to glycopyrronium (this difference was significant) and by $10 \%$ when compared to open-labelled tiotropium (not significant). A more pronounced effect was found regarding mild exacerbations, and was significant versus both glycopyrronium and tiotropium. The clinical significance of this finding is not elucidated, although similar differences were found regarding health status [6].

\section{Rationale for bronchodilator combinations in COPD}

While bronchodilators are considered the cornerstone of COPD pharmacological treatment, there is a clear pharmacological rationale for combining $\beta_{2}$-agonists and anti-muscarinic agents, as recently reviewed [7].

Received: Sept 262013 | Accepted: Sept 262013

Conflict of interest: Disclosures can be found alongside the online version of this article at www.erj.ersjournals.com 
Airway smooth muscle cells (ASM) express $\beta_{2}$-adrenoreceptors that respond to their agonists by relaxing the smooth muscle, and anticholinergic agents block their receptors on ASM, preventing them from being activated by acetylcholine. Thus, additive effects could be expected, resulting in an increase in 1) the likelihood of obtaining bronchodilation and 2) the magnitude of effects on lung function. Furthermore, interactions between adrenergic and cholinergic pathways could lead to synergistic effects. Despite these observations, the nature (additive or synergistic) of the net clinical result obtained by co-administration of $\beta_{2}$-agonists and anticholinergics is not completely elucidated from a pharmacological perspective.

Initial studies assessing co-administration using short-acting agents found that the effect of the two classes combined on lung function was greater than could be obtained with a single agent [7]. An additional reduction in exacerbation rate was also reported. Similarly, additional effects on lung function and symptom scores were observed when combining agents of different durations of action (SABA+LAMA or LABA+SAMA). Finally, the acute and chronic co-administration of a LABA and a LAMA in separate inhalers was shown to provide greater bronchodilation than either agent alone. In addition, some studies found that many outcomes such as symptoms, health status and use of rescue bronchodilators were favourably affected by this combined strategy. While the differences observed were usually statistically significant, their clinical relevance is unclear since they did not reach thresholds of "minimal clinically important differences". As a consequence, most guidelines recommend to associate two long-acting bronchodilators only when one does not relieve symptoms, rather than as a first-line option [8,9]. Notably, all studies assessing this combination approach reported an overall excellent safety profile.

Altogether, it appears coherent to develop combinations of long-acting bronchodilators to improve efficacy (with, at least, additive effects) and practicability (one single inhaler, once daily administration, both potentially favouring adherence to treatment) without increasing side-effects.

\section{What can we expect from a combination of bronchodilators in COPD?}

When new pharmacological treatments or combinations for COPD are evaluated in clinical trials, a frequent criticism is that the magnitude of effects on clinical outcomes is of relatively low magnitude. Such statements need to be put into the perspective of the disease. COPD is a multifaceted disease with a mixture of airway and parenchymal disease, proximal and distal airway involvement and reversible and irreversible components.

Dyspnoea is the major symptom and plays a prominent role in health status impairment. Lung hyperinflation is a dominant determinant of dyspnoea and bronchodilators primarily aim at reducing its level through decreasing airflow limitation. Bronchodilators have also been shown to reduce the frequency of exacerbations through debated mechanisms [9].

In COPD, airflow limitation is largely due to airway structural changes that have major consequences on airway resistance, especially where small airways are concerned [10]. In addition, emphysema, which is preceded by bronchiolar loss [11], contributes to increasing small airways resistance. These phenomena are largely irreversible. Therefore, the beneficial effect of bronchodilators is intrinsically limited by the nature of the disease and the mean magnitude of differences between one and two bronchodilators cannot be huge. We should keep this in mind when interpreting results of RCTs, although it does not mean that these agents are useless: they have been shown to provide improvements in lung function, dyspnoea perception, exercise tolerance, health status and prevention of exacerbations $[8,12]$.

Reaching the minimal clinically important difference: a dream or an achievable goal? In the present highly constrained economical context, new treatments are scrutinised by health authorities with increasing intensity. The reality of increased efficacy should be demonstrated against each component with a favourable safety profile. The current Global Initiative for Chronic Obstructive Lung Disease (GOLD) document does not really propose a progressive stepwise (first- then second-line) strategy, but only mentions that the choice between LABA, LAMA and LABA+LAMA has to be made considering individual treatment response, and that LABA+LAMA combinations are alternative options for the treatment of GOLD B, C and D stages. National Institute for Health and Clinical Excellence guidelines recommend a LABA, LAMA or inhaled corticosteroid (ICS)/LABA as first option, depending on FEV1 and symptoms. LABA+LAMA combinations are considered only as an alternative to ICS/LABA when ICS treatment is declined or not tolerated [9]. Few clinical trials have assessed single-device combined bronchodilator therapy to define where these treatments should stand in treatment guidelines.

Although FEV1 is still "alive", it is only a prerequisite for assessment of bronchodilators' efficacy and effectiveness: in other words, it is certainly not a valuable single end-point in COPD. Studies were conducted to establish the so-called "thresholds of clinical significance" or "minimal clinically important difference" (MCID), i.e. to determine which magnitude of effect is required for patients to perceive a 
benefit. Some of these studies proposed FEV1 thresholds to distinguish clinically relevant versus clinically irrelevant variations in this variable [13]. Although these thresholds may be of some value to evaluate the chance of obtaining relevant clinical effects, their value to predict such effects is limited. Indeed, giving too much weight to FEV1 thresholds is somewhat schizophrenic, since the poor association between treatment effects on airflow obstruction and clinical outcomes (dyspnoea, health status) is well demonstrated. Thus, developing MCIDs for clinical variables appears more relevant $[14,15]$. Methods to explore MCIDs can follow two approaches: anchoring and statistics. Basically, anchoring methods rely on the relationships between changes in the variable of interest and one or several reference variables, i.e. variables that already have an established MCID or Likert-like scales asking the patient or healthcare professional to grade how much of a change they perceive. The statistical approach is based on the distribution of the values recorded in the population of interest. It must be outlined that none of these methods is completely satisfactory since results are influenced by several factors, including population characteristics (e.g. disease severity, reversibility and concomitant treatments), chosen reference variables and treatment duration. Therefore, conclusions must remain cautious. Overall, it is probably preferable (i.e. more clinically relevant, especially from an individual perspective) to express results in terms of proportion of patients in which the treatment effect reaches the MCID than in terms of mean treatment effect. In the SHINE study, the effect of QVA149 on transition dyspnoea index (TDI) was 0.51 points higher than that of tiotropium: on the one hand, this is only half of TDI's MCID (1 point). But on the other hand, the proportion of patients whose TDI improved by more than 1 point was roughly 10\% higher (absolute increase) with QVA149 than with tiotropium (15\% relative increase, which may be interesting from an individual clinical perspective).

Finally, three characteristics of QVA149 could favour adherence to treatment, thus prompting integration in treatment algorithms: once-daily dosing, presence of two agents in one device and fast onset of action [16]. However, the real impact of combining these characteristics on adherence and treatment effectiveness in COPD patients remains to be formally assessed.

\section{Should a specific COPD phenotype be targeted?}

It has long been known that COPD is a heterogeneous disease, comprising patients with various degrees of bronchial versus emphysematous profiles [17]. In the present era of P4 medicine (personalised, predictive, preventive and participatory) [18], identifying phenotypes associated with response to treatments would definitely be of clinical interest in order to help individualise therapy. The last versions of the GOLD document identify patients according to symptoms and risk of exacerbations, as reflected by FEV1 level and history of exacerbations. GOLD stages are associated with differences in prognosis, health status, exacerbation risk and frequency of comorbidities [19]. How they relate to response to treatment and how they can be modified by treatments warrant future investigations.

Numerous possible predictors of response to bronchodilator treatments have been investigated including age, sex, smoking status, severity of airflow obstruction, acute reversibility to bronchodilators, previous history of exacerbations and concomitant treatments (inhaled corticosteroids); most studies actually failed to identify subgroups of more significant responders [20-22]. Accordingly, BATEMAN et al. [2] did not find any significant difference in lung function improvement according to age, sex and ICS use. Although lung function improved more in patients with moderate versus severe airflow obstruction, the effect was still statistically and clinically significant in those with severe airflow obstruction.

It has been hypothesised that patients with acute reversibility to bronchodilators may respond clinically to a long-term treatment. However, several studies found that reversibility is variable in a given patient and does not predict long-term outcomes, leading authors to consider it as an "unreliable phenotype" [23]. Another approach could be to assess bronchodilator effects in bronchial versus emphysematous phenotypes, hypothesising that such treatments are less likely to exert beneficial effects when patients have a more "destructive" than "constrictive" disease. High-resolution computed tomography (HRCT) scan, resting lung volumes and diffusing capacity of the lung for carbon monoxide are of potential interest to facilitate recognition these phenotypes. HRCT scan is able to investigate airway wall sizes and emphysema, which are inversely related in severe COPD [24]. However, data on the relationship of these CT indices with clinical features such as sputum production, bronchodilator reversibility or exacerbations are contradictory [25-28]. Increased resting hyperinflation has been associated with more bronchodilator-induced acute volume deflation [29], decreasing exercise-induced dyspnoea [30], but its predictive value at an individual level has not been investigated. Low transfer factor has been associated with decreased acute response to bronchodilators [31], but has seldom been studied as a predictor of long-term effects [32]. Finally, airway inflammation phenotype is linked to acute bronchodilator response, which is greater when eosinophilic inflammation is present [33]. 
In summary, identification of clinical responders to bronchodilators is an unsolved challenge at present. While the study by BATEMAN et al. [2] addresses the effect of combination versus single bronchodilation, an important additional issue is the positioning of dual bronchodilation versus ICS-LABA combinations in the COPD therapeutic strategy: some phenotypes could be associated with a predominant response to one of these combinations or the other. Defining the respective roles of these agents requires head to head comparisons investigating exacerbations but also dyspnoea, exercise tolerance and health status. In patients with moderate-to-severe COPD and no history of exacerbations, QVA149 has been shown to improve lung function and dyspnoea more than salmeterol-fluticasone combination, with no significant difference in terms of health status [34]. Further studies are required to explore how these approaches compare in previously exacerbating patients.

\section{Key messages}

The magnitude of the effect of any pharmacological treatment is intrinsically limited in COPD. This does not mean that these approaches are useless. Some may consider that contenting ourselves with limited treatment effects is not a cost-effective but rather a compassionate attitude. This might be true, but providing patients with some relief might also be viewed as a respectable compassionate attitude, considering the overall COPD burden in terms of handicap.

On the same line, results of clinical trials have to be interpreted with caution regarding the concept of "minimal clinically important difference", since many factors may influence MCID thresholds.

A research strategy should be developed to better dismantle the conditions ranged under the umbrella of COPD and then better understand the potential dedicated management for a given patient. This strategy, despite its cost and uncertainty, should be used at an early stage of an innovative treatment development.

\section{References}

1 Cazzola M, Page CP, Calzetta L, et al. Emerging anti-inflammatory strategies for COPD. Eur Respir J 2012; 40: 724-741.

2 Bateman ED, Ferguson GT, Barnes N, et al. Dual bronchodilation with QVA149 versus single bronchodilator therapy: the SHINE study. Eur Respir J 2013; 42: 1484-1494.

3 Dahl R, Chapman KR, Rudolf M, et al. Safety and efficacy of dual bronchodilation with QVA149 in COPD patients: the ENLIGHTEN study. Respir Med 2013; 107: 1558-1567.

4 Van Noord JA, Buhl R, Laforce C, et al. QVA149 demonstrates superior bronchodilation compared with indacaterol or placebo in patients with chronic obstructive pulmonary disease. Thorax 2010; 65: 1086-1091.

5 Van de Maele B, Fabbri LM, Martin C, et al. Cardiovascular safety of QVA149, a combination of Indacaterol and NVA237, in COPD patients. COPD 2010; 7: 418-427.

6 Wedzicha JA, Decramer M, Ficker JH, et al. Analysis of chronic obstructive pulmonary disease exacerbations with the dual bronchodilator QVA149 compared with glycopyrronium and tiotropium (SPARK): a randomised, doubleblind, parallel-group study. Lancet Respir Med 2013; 1: 199-209.

7 Cazzola M, Molimard M. The scientific rationale for combining long-acting beta2-agonists and muscarinic antagonists in COPD. Pulm Pharmacol Ther 2010; 23: 257-267.

8 Global Initiative for Chronic Obstructive Lung Disease. Global Strategy for the Diagnosis, Management and Prevention of Chronic Obstructive Lung Disease. 2013. Available from www.goldcopd.org

9 National Institute for Health and Clinical Excellence. CG101 Chronic Obstructive Pulmonary Disease (Update): Full Guideline. London, Royal College of Physicians/NICE, 2010.

10 Burgel PR, Bourdin A, Chanez P, et al. Update on the roles of distal airways in COPD. Eur Respir Rev 2011; 20: $7-22$.

11 McDonough JE, Yuan R, Suzuki M, et al. Small-airway obstruction and emphysema in chronic obstructive pulmonary disease. N Engl J Med 2011; 365: 1567-1575.

12 Wedzicha JA, Decramer M, Seemungal TAR. The role of bronchodilator treatment in the prevention of exacerbations of COPD. Eur Respir J 2012; 40: 1545-1554.

13 Donohue JF. Minimal clinically important differences in COPD lung function. COPD 2005; 2: 111-124.

14 Jones PW, Bosh TK. Quality of life changes in COPD patients treated with salmeterol. Am J Respir Crit Care Med 1997; 155: 1283-1289.

15 Witek TJ, Mahler DA. Minimal important difference of the transition dyspnoea index in a multinational clinical trial. Eur Respir J 2003; 21: 267-272.

16 Charles MS, Blanchette CM, Silver $\mathrm{H}$, et al. Adherence to controller therapy for chronic obstructive pulmonary disease: a review. Curr Med Res Opin 2010; 26: 2421-2429.

17 Burrows B. The bronchial and emphysematous types of chronic obstructive lung disease in London and Chicago. Aspen Emphysema Conf 1968; 9: 327-338.

18 Agusti A, Sobradillo P, Celli B. Addressing the complexity of chronic obstructive pulmonary disease: from phenotypes and biomarkers to scale-free networks, systems biology, and P4 medicine. Am J Respir Crit Care Med 2011; 183: 1129-1137.

19 Agusti A, Hurd S, Jones P, et al. FAQs about the GOLD 2011 assessment proposal of COPD: a comparative analysis of four different cohorts. Eur Respir J 2013; 42: 1391-1401.

20 Celli B, Vestbo J, Jenkins CR, et al. Sex differences in mortality and clinical expressions of patients with chronic obstructive pulmonary disease: the TORCH experience. Am J Respir Crit Care Med 2011; 183: 317-322. 
21 Jenkins CR, Jones PW, Calverley PM, et al. Efficacy of salmeterol/fluticasone propionate by GOLD stage of chronic obstructive pulmonary disease: analysis from the randomised, placebo-controlled TORCH study. Respir Res 2009; 10: 59.

22 Tashkin DP, Celli B, Kesten S, et al. Long-term efficacy of tiotropium in relation to smoking status in the UPLIFT trial. Eur Respir J 2010; 35: 287-294.

23 Albert P, Agusti A, Edwards L, et al. Bronchodilator responsiveness as a phenotypic characteristic of established chronic obstructive pulmonary disease. Thorax 2012; 67: 701-708.

24 Kim WJ, Silverman EK, Hoffman E, et al. CT metrics of airway disease and emphysema in severe COPD. Chest 2009; 136: 396-404.

25 Tsushima K, Sone S, Yoshikawa S, et al. Clinical differences in the Global Initiative for Chronic Obstructive Lung Disease Stage 0. Respir Med 2006; 100: 1360-1367.

26 Lee JS, Huh JW, Chae EJ, et al. Response patterns to bronchodilator and quantitative computed tomography in chronic obstructive pulmonary disease. Clin Physiol Funct Imaging 2012; 32: 12-18.

27 Han MK, Bartholmai B, Liu LX, et al. Clinical significance of radiologic characterizations in COPD. COPD 2009; 6: 459-467.

28 Han MK, Kazerooni EA, Lynch DA, et al. Chronic obstructive pulmonary disease exacerbations in the COPDGene study: associated radiologic phenotypes. Radiology 2011; 261: 274-282.

29 Deesomchok A, Webb KA, Forkert L, et al. Lung hyperinflation and its reversibility in patients with airway obstruction of varying severity. COPD 2010; 7: 428-437.

30 O’Donnell DE, Lam M, Webb KA. Spirometric correlates of improvement in exercise performance after anticholinergic therapy in chronic obstructive pulmonary disease. Am J Respir Crit Care Med 1999; 160: 542-549.

31 Izquierdo-Alonso JL, Sánchez-Hernández I, Fernández Francés J, et al. Utility of transfer factor to detect different bronchodilator responses in patients with chronic obstructive pulmonary disease. Respir Int Rev Thorac Dis 1998; 65: 282-288.

32 Terzano C, Petroianni A, Ricci A, et al. Combination therapy in COPD: different response of COPD stages and predictivity of functional parameters. Eur Rev Med Pharmacol Sci 2005; 9: 209-215.

33 Perng DW, Huang HY, Chen HM, et al. Characteristics of airway inflammation and bronchodilator reversibility in COPD: a potential guide to treatment. Chest 2004; 126: 375-381.

34 Vogelmeier CF, Bateman ED, Pallante J, et al. Efficacy and safety of once-daily QVA149 compared with twice-daily salmeterol-fluticasone in patients with chronic obstructive pulmonary disease (ILLUMINATE): a randomised, double-blind, parallel group study. Lancet Respir Med 2013; 1: 51-60. 